\title{
Psychological Impacts of the COVID-19 Pandemic on One-month Postpartum Mothers: A Retrospective Study from a Consecutive Database in a Metropolitan Area of Japan
}

\author{
Youji Takubo \\ Department of Neuropsychiatry, Toho University Graduate School of Medicine \\ Naohisa Tsujino ( $\nabla$ ntsujino@med.toho-u.ac.jp) \\ Department of Psychiatry, Saiseikai Yokohamashi Tobu Hospital \\ Yuri Aikawa \\ Department of Psychiatry, Saiseikai Yokohamashi Tobu Hospital \\ Kazuyo Fukiya \\ Department of Psychiatry, Saiseikai Yokohamashi Tobu Hospital \\ Momoko Iwai \\ Department of Neuropsychiatry, Toho University Faculty of Medicine \\ Takashi Uchino \\ Department of Neuropsychiatry, Toho University Faculty of Medicine \\ Megumu Ito \\ Department of Obstetrics and gynecology, Saiseikai Yokohamashi Tobu Hospital \\ Yasushi Akiba \\ Department of Obstetrics and gynecology, Saiseikai Yokohamashi Tobu Hospital \\ Masafumi Mizuno \\ Tokyo Metropolitan Matsuzawa Hospital \\ Takahiro Nemoto \\ Department of Neuropsychiatry, Toho University Faculty of Medicine
}

\section{Research Article}

Keywords: Anxiety, COVID-19, Japan, Postnatal Depression, Postpartum

Posted Date: July 20th, 2021

DOI: https://doi.org/10.21203/rs.3.rs-659189/v1

License: (c) (1) This work is licensed under a Creative Commons Attribution 4.0 International License. Read Full License 


\section{Abstract}

\section{Background:}

The coronavirus disease 2019 (COVID-19) pandemic has recently become the most important issue in the world. Very few reports in Japan have examined the impact of the COVID-19 pandemic on peripartum mental health. We examined the current status of postpartum mental health in a metropolitan area.

\section{Methods:}

The subjects were women who had completed a maternity health check-up at a core regional hospital in Yokohama during the period from April 1, 2017, to December 31, 2020. We collected the subjects' scores for the Edinburgh Postnatal Depression Scale (EPDS) and the Mother-to-Infant Bonding Scale (MIBS) at one month postpartum. The subjects were divided into four groups (three Before COVID-19 groups and a During COVID-19 group). MANOVA and post-hoc tests were used to determine mental health changes in the postpartum period among the four groups.

\section{Results:}

The Before and During COVID-19 groups contained 2844 and 1095 mothers, respectively. There were no significant difference in the total scores of the EPDS and MIBS among the four groups. However, the EPDS items related to anxiety factors were significantly higher and the EPDS items related to anhedonia and depression factors (excluding thoughts of self-harm) were significantly lower in the During COVID-19 group.

\section{Conclusion:}

The EPDS scores changed in connection with the COVID-19 pandemic. Anxiety, which represent hypervigilance, was significantly higher and anhedonia and depression were significantly lower in the During COVID-19 group. Our results may reflect COVID-19-related health concerns and a lack of social support caused by the COVID-19 pandemic.

\section{Background}

The coronavirus disease 2019 (COVID-19) pandemic has recently become the most important issue in the world, and no one has been unaffected by its impact [1]. The first known cases occurred in China in December 2019, and the first Japanese case in a traveler returning from China was reported on January 16, 2020, in Kanagawa Prefecture, which is adjacent to the Tokyo Metropolitan area [2]. The World Health Organization (WHO) declared COVID-19 to be a global pandemic on March 11, 2020 [3]. The Government of Japan issued a statement of emergency in seven major areas, including Kanagawa Prefecture, on April 7, 2020, and expanded the target area nationwide on April 16 [4]. Fear and worry about COVID-19, the impact of the statement of emergency on daily life, and the difficulty in predicting the situation caused much stress and led to mental health problems. Some evidence of COVID-19 and its relation to mental health issues has been published in different countries, and the psychological impact of COVID-19 is already obvious, both in the general population and in people with existing mental disorders $[5,6]$. Previous studies have shown that the COVID-19 epidemic has caused a sharp increase in the prevalence of anxiety and depressive disorders among the general adult population in the world [7-11]. In particular, people aged 18 to 34 years, women, and people living with infants are more vulnerable to COVID-19-related stress $[5,12]$. A Japanese study also revealed that individuals who were in young age groups and in economically vulnerable situations showed worse mental health during the COVID-19 crisis [13]. These studies suggest that young women who live with infants may be susceptible to mental distress during the COVID-19 pandemic.

Mental illnesses, such as depressive and anxiety disorders, are among the most common morbidities during pregnancy and in the postpartum period, and the existence of mental distress in mothers can have adverse effects on the psychological development of their children [14-17]. Concern over COVID-19 causing critical illness or death is at the core of everyone's anxiety. In addition, mothers may be worried more about their children than themselves. The impact of the COVID-19 pandemic on the mental health of perinatal mothers has also been clarified $[18,19]$. Some studies have demonstrated high rates of stress, anxiety, and depressive symptoms, including thoughts of self-harm, in pregnant and postpartum mothers around the world during the COVID-19 pandemic [20-27]. A systematic review and meta-analysis of eight studies using the Edinburgh Postnatal Depression Scale (EPDS) and the State-Trait Anxiety Inventory (STAI) examined the impact of the COVID-19 pandemic on anxiety and depression in pregnancy and the perinatal period [26]. In that study, the effect sizes and standardized mean differences (SMDs) and the corresponding 95\% confidence intervals (Cls) were calculated using the random-effects model. Whereas the EPDS score did not reach a statistically significant difference, the STAI score was significantly higher during the pandemic than in previous non-pandemic times [26]. In addition, a study of 2740 pregnant women during the pandemic found that stopping face-to-face prenatal visits and modifications to birth plans were strongly associated with anxiety [24]. Similarly, previous studies have suggested that social isolation and quarantine exacerbated depression and anxiety among pregnant women [21, 28].

Regarding mother-to-infant bonding, maternal bonding is the creation of an emotional bond between a mother and her newborn baby, and bonding failure is characterized by an aversion to the infant and a marked impairment in interactions, including decreased maternal affection, increased irritability, and rejection of the infant [29]. Impaired bonding has been suggested to lead to maltreatment and child abuse [29]. The coexistence of bonding failure and postpartum depression has been reported, although a causal relationship remains unclear [30, 31]. Very few studies have evaluated the impact of the COVID-19 pandemic on mother-to-infant bonding [32].

The worldwide COVID-19 pandemic is ongoing in Japan, especially in the Tokyo metropolitan area. However, very few reports have examined the impacts of the COVID-19 pandemic on peripartum depressive and anxious symptoms and mother-to-infant bonding in Japan [33, 34]. While an online EPDS-based survey of pregnant women during the COVID-19 pandemic was conducted in Japan, the respondents were not compared with a control group that reflected the situation before the COVID-19 pandemic [34]. The total number of people infected with COVID-19 in Kanagawa Prefecture, which is part of the Tokyo 
metropolitan area, was 61,516 at the end of May 2021 [35]. Kanagawa is located next to Tokyo, which is the area of Japan with the highest number of total COVID-19 cases. To the best of our knowledge, no data on the impact of the COVID-19 pandemic on mental health in postpartum women has been reported in Japan to date; specifically, no comparisons with a control group that reflects the situation before the COVID-19 pandemic have been made. To provide integrated postpartum treatment during the COVID-19 era, an examination of the current status of postpartum mental health, such as depressive and anxious symptoms as well as mother-to-infant bonding, is needed. Accordingly, the aim of the present study was to investigate the current status of postpartum mothers' mental health taking into consideration the influence of the COVID-19 pandemic in a metropolitan area of Japan by comparing the EPDS and Mother to Infant Bonding Scale (MIBS) scores of mothers before and during the COVID-19 pandemic.

\section{Methods \\ Procedures and subjects}

This study consisted of a retrospective chart review that was aimed at examining the influence the COVID-19 pandemic on women's mental health at one month postpartum. The Saiseikai Yokohamashi Tobu Hospital is a regional core general hospital that covers the eastern area of Yokohama City in Kanagawa Prefecture; it also accepts people with COVID-19. For the early detection and intervention of postpartum mental health problems and the identification of their causes, all women are asked to complete the Japanese versions of the EPDS and MIBS at the time of a one-month maternity health check-up, and a continuous hospital database of this information has been maintained since April 1, 2017. The subjects were women who gave birth and had a maternity health check-up at one month after childbirth in the Obstetrics and Gynecology Departments of the Saiseikai Yokohamashi Tobu Hospital, which also has a continuous database. We set the investigation period as lasting from April 1, 2017, to December 31, 2020. The EPDS and MIBS scores at one month postpartum and the subjects' demographic and obstetric information, including the weeks of childbirth, delivery style, parity, and birth weight of the child, were collected from the continuous database and medical charts.

The primary outcome consisted of the EPDS and MIBS scores. Cox et al. developed the EPDS, and the Japanese version was reported by Okano to have a good internal consistency [36, 37]. Taylor et al. developed the MIBS, and the Japanese version was translated and validated by Yoshida [38, 39]. Both the EPDS and the MIBS are self-reported instruments with 10 items rated on a 4-point scale with total scores ranging from 0 to 30 [36-39]. The higher the score of EPDS and MIBS, the worse the symptoms of postpartum depression and mother-to-infant bonding, respectively. In the Japanese version of the EPDS, the optimal cut-off score was 8/9 for screening for perinatal depression [37]. The EPDS has been suggested to have a two- or three-factor structure. A recent Japanese study using a large dataset revealed a three-factor structure model consisting of "Anhedonia" (Items 1 and 2), "Anxiety" (Items 3, 4, 5, and 6), and "Depression" (Items 7, 9, and 10). This model demonstrated a high goodness-of-fit [40]. On the other hand, a Japanese study using a cluster analysis suggested the presence of mothers with pathological maternal bonding (14.4\%), with an optimal MIBS cut-off score of 4/5 at one month after delivery [41]. The MIBS was suggested to have a two-factor structure composed of "Lack of affection" (Items 1, 6, 8, and 10) and "Anger and rejection" (Items 2, 3, 5, and 7) [39]. We used the above cut-off scores and factor structures in the present analysis.

This study was performed as part of the Mental health and Early Intervention in the Community-based Integrated care System (MEICIS) project, which is supported by a Health Labour Sciences Research Grant (19GC1015).

The study protocol was approved by the Ethics Committees of Saiseikai Yokohamashi Tobu Hospital (20200129). Informed consent was obtained in the form of opt-out on a website. The study was performed in accordance with the latest version of the Declaration of Helsinki (October 2013).

\section{Statistical analysis}

The subjects were divided into two groups based on a cut-off date of January 16, 2020, when the first case of COVID-19 was reported in Japan. Subjects who first visited the Department of Psychiatry during the period from April 1, 2017, to January 15, 2020, were included in the "Before COVID-19" group, while those who first visited during the period from January 16, 2020, to December 31, 2020, were included in the "During COVID-19" group.

After comparing demographic and clinical characteristics among the two groups, we divided the Before COVID-19 group into three subgroups according to year (2017: April 1, 2017, to December 31, 2017; 2018: January 1, 2018, to December 31, 2018; 2019: January 1, 2019, to January 15, 2020). To examine the impact of the COVID-19 pandemic more precisely, we then compared each primary outcome among the four groups (the three Before COVID-19 subgroups and the During COVID-19 group [2020: January 16, 2020, to December 31, 2020]). We used a multivariate analysis of variance (MANOVA) followed by post-hoc Tukey's honestly significant difference (HSD) and chi-square tests to compare demographic and clinical variables (such as age, parity, and delivery style; and the total scores of EPDS and MIBS) between the groups. The Kruskal-Wallis test was used to test for the proportion of postpartum depression or postpartum pathological maternal bonding among the groups. All the statistical analyses were conducted using IBM SPSS, version 26.0.

\section{Results}

A total of 3985 participants (2880 mothers in the Before COVID-19 group and 1105 mothers in the During COVID-19 group) were included. Thirty-six mothers in the Before COVID-19 group and 10 mothers in the During COVID-19 group were excluded because they did not complete the EPDS or the MIBS questionnaire. The proportion of women excluded in the analysis was small (1.2\%). The study flow chart is shown in Fig. 1.

The mean age of all the participants was $33.4(\mathrm{SD}=5.1)$ years. The proportions of the participants who were under 34 years old, were primipara, had a fullterm birth (> 38 weeks), delivered vaginally, and had a singleton live birth were $57.9 \%, 48.5 \%, 95.9 \%, 71.8 \%$, and $97.9 \%$, respectively. Regarding the birth weight of the infant, $88.3 \%$ of the infants were of normal weight. None of the mothers in this study contracted COVID-19 during pregnancy or the postpartum period. 
The general and obstetric characteristics of the participants are shown in Table 1. A t-test and the chi-square test showed no significant difference in general and obstetric characteristics between the Before COVID-19 and the During COVID-19 groups.

Table 1 General and obstetric characteristics of subjects before and during the COVID-19 pandemic

\begin{tabular}{|c|c|c|c|c|c|c|}
\hline & \multicolumn{2}{|c|}{ Before COVID-19 } & \multicolumn{2}{|c|}{ During COVID-19 } & \multirow[b]{2}{*}{ Statistics } & \multirow[b]{2}{*}{$p$} \\
\hline & $\mathrm{N}$ & $\%$ & $\mathrm{~N}$ & $\%$ & & \\
\hline \multicolumn{7}{|l|}{ Age } \\
\hline Mean (SD) & 33.4 & 5.1 & 33.2 & 5.2 & $\mathrm{t}=1.186$ & 0.236 \\
\hline$<34$ & 1636 & $57.5 \%$ & 645 & $58.9 \%$ & \multirow[t]{2}{*}{$\chi^{2}=0.617$} & \multirow[t]{2}{*}{0.432} \\
\hline$\geqq 35$ & 1208 & $42.5 \%$ & 450 & $41.1 \%$ & & \\
\hline \multicolumn{7}{|l|}{ Parity } \\
\hline Primipara & 1390 & $48.9 \%$ & 520 & $47.5 \%$ & \multirow{2}{*}{$x^{2}=0.608$} & \multirow[t]{2}{*}{0.435} \\
\hline Multipara & 1454 & $51.1 \%$ & 575 & $52.5 \%$ & & \\
\hline \multicolumn{7}{|l|}{ Birth weeks } \\
\hline Premature delivery (<37weeks) & 119 & $4.2 \%$ & 44 & $4.0 \%$ & \multirow[t]{2}{*}{$\chi^{2}=0.055$} & \multirow[t]{2}{*}{0.815} \\
\hline Full term birth ( $\geqq 38$ weeks) & 2725 & $95.8 \%$ & 1051 & $96.0 \%$ & & \\
\hline \multicolumn{7}{|l|}{ Delivery style } \\
\hline Vaginal delivery & 2034 & $71.5 \%$ & 793 & $72.4 \%$ & \multirow[t]{2}{*}{$\chi^{2}=0.317$} & \multirow[t]{2}{*}{0.574} \\
\hline Caesarean Section & 810 & $28.5 \%$ & 302 & $27.6 \%$ & & \\
\hline \multicolumn{7}{|l|}{ Birth } \\
\hline Single birth & 59 & $2.1 \%$ & 22 & $2.0 \%$ & \multirow[t]{2}{*}{$x^{2}=0.017$} & \multirow[t]{2}{*}{0.897} \\
\hline Multiple birth & 2785 & $97.9 \%$ & 1073 & $98.0 \%$ & & \\
\hline \multicolumn{7}{|l|}{ Birth weight of infant } \\
\hline Normal or high birth weight infant ( $\geqq 2500 \mathrm{~g})$ & 2560 & $88.2 \%$ & 989 & $88.5 \%$ & \multirow[t]{2}{*}{$\chi^{2}=0.099$} & \multirow[t]{2}{*}{0.753} \\
\hline Low birth weight infant $(<2500 \mathrm{~g})$ & 343 & $11.8 \%$ & 128 & $11.5 \%$ & & \\
\hline
\end{tabular}

The Before COVID-19 group was also divided into three subgroups according to year (2017: $n=706 ; 2018: n=1142 ; 2019: n=996)$. We compared the average EPDS and MIBS scores for the three Before subgroups plus the During COVID-19 group $(2020: n=1095)$. Comparisons of the scores for each item using the MANOVA are shown in Tables 2 . The analysis revealed a significant overall difference between the four groups (Wilks' lambda $=0.379, F(60,11684)=74.6, p$ $<0.001$ ) for the primary outcomes.

The average EPDS scores for each item in the 2017, 2018 and 2019 subgroups were statistically consistent, and only those for 2020 differed significantly (except for EPDS Item 10). The scores for Items 3, 4, 5, and 6 were significantly higher during COVID-19, while the scores for Items 1, 2, 7, 8, and 9 were significantly lower during COVID-19. Regarding the factor structure, the sum score for "EPDS anxiety $(\operatorname{Items} 3,4,5$, and 6$)$ " $\left[F=643.728, p<0.001, \eta^{2}=0.329\right]$ was significantly higher during COVID-19, while the sum scores for "EPDS anhedonia (Items 1, and 2)" $\left[F=249.236, p<0.001, \eta^{2}=0.160\right]$ and "EPDS depression (Items 7, 9, and 10)" $\left[F=95.415, p<0.001, \eta^{2}=0.068\right]$ were significantly lower during COVID-19.

On the other hand, the score for MIBS Item 6 showed significant difference between the four groups, whereas no significant differences in the other MIBS items were seen. Post hoc comparison for the score for MIBS Item 6 yielded significant difference in 2017 vs. 2020 ( $p=0.023)$, and 2019 vs. 2020 ( $p=0.025$ ), however there were no significant difference in 2018 vs. 2020 ( $p=0.948)$. Comparisons of each item in the factor structure are shown in Figs. $2 a$ (EPDS, Anhedonia items), 2b (EPDS Anxiety items), 2c (EPDS, Depression items), 3a (MIBS, Lack of affection items), and 3b (MIBS, Anger and rejection items).

No significant difference in the average total scores of the EPDS $\left(2017: 4.72 ; 2018: 4.59 ; 2019: 4.71 ;\right.$ and $\left.2020: 4.70 ; F=0.247, p=0.864, \eta^{2}=<0.001\right)$ or the MIBS (2017: 2.04; 2018: 1.94; 2019: 1.84; and 2020: 1.84; $F=1.587 p=0.190, \eta^{2}=0.001$ ) was seen, using a one-way Analysis of variance (one-way ANOVA).

Table 2 Comparison of EPDS and MIBS scores for each item 


\begin{tabular}{|c|c|c|c|c|c|c|c|c|c|c|c|c|c|c|c|c|c|}
\hline & & & & & & & & & & & & \multicolumn{6}{|c|}{ Post-hoc (Tukey's HSD) } \\
\hline & \multicolumn{2}{|l|}{2017} & \multicolumn{2}{|l|}{2018} & \multicolumn{2}{|l|}{2019} & \multicolumn{2}{|l|}{2020} & \multirow{2}{*}{\multicolumn{3}{|c|}{ MANOVA }} & \multirow{2}{*}{$\begin{array}{l}2017 \\
\text { vs } \\
2018\end{array}$} & \multirow{2}{*}{$\begin{array}{l}2017 \\
\text { vs } \\
2019\end{array}$} & \multirow{2}{*}{\multicolumn{2}{|c|}{$\begin{array}{l}2017 \text { vs } \\
2020\end{array}$}} & \multirow{2}{*}{$\begin{array}{l}2018 \\
\text { vs } \\
2019\end{array}$} & \multirow{2}{*}{$\begin{array}{l}2018 \text { vs } \\
2020\end{array}$} \\
\hline & $n=706$ & & $\mathrm{n}=1142$ & & $\mathrm{n}=996$ & & $\mathrm{n}=1095$ & & & & & & & & & & \\
\hline & Mean & SD & Mean & SD & Mean & SD & Mean & SD & $\mathrm{F}$ & $p$ & & $p$ & $p$ & $p$ & & $p$ & $p$ \\
\hline EPDS1 & 0.98 & 0.87 & 0.96 & 0.88 & 0.93 & 0.89 & 0.07 & 0.29 & 351.607 & 0.000 & $* *$ & 0.961 & 0.590 & 0.000 & ** & 0.813 & 0.000 \\
\hline EPDS2 & 0.45 & 0.73 & 0.41 & 0.70 & 0.45 & 0.74 & 0.16 & 0.44 & 48.756 & 0.000 & $* *$ & 0.501 & 1.000 & 0.000 & ** & 0.352 & 0.000 \\
\hline EPDS3 & 0.24 & 0.52 & 0.22 & 0.52 & 0.27 & 0.57 & 0.95 & 0.88 & 312.737 & 0.000 & ** & 0.905 & 0.758 & 0.000 & ** & 0.242 & 0.000 \\
\hline EPDS4 & 0.24 & 0.48 & 0.20 & 0.46 & 0.24 & 0.48 & 0.86 & 0.91 & 271.466 & 0.000 & ** & 0.747 & 1.000 & 0.000 & ** & 0.642 & 0.000 \\
\hline EPDS5 & 0.09 & 0.31 & 0.09 & 0.34 & 0.09 & 0.34 & 0.46 & 0.75 & 149.236 & 0.000 & $\star *$ & 0.985 & 0.994 & 0.000 & ** & 1.000 & 0.000 \\
\hline EPDS6 & 0.12 & 0.36 & 0.13 & 0.42 & 0.18 & 0.49 & 1.13 & 0.80 & 812.361 & 0.000 & ** & 0.928 & 0.128 & 0.000 & ** & 0.273 & 0.000 \\
\hline EPDS7 & 0.92 & 0.94 & 0.87 & 0.91 & 0.87 & 0.91 & 0.26 & 0.58 & 145.689 & 0.000 & ** & 0.576 & 0.665 & 0.000 & ** & 0.999 & 0.000 \\
\hline EPDS8 & 1.16 & 0.82 & 1.19 & 0.79 & 1.17 & 0.82 & 0.50 & 0.70 & 196.613 & 0.000 & $* *$ & 0.871 & 1.000 & 0.000 & ** & 0.889 & 0.000 \\
\hline EPDS9 & 0.47 & 0.65 & 0.46 & 0.67 & 0.44 & 0.66 & 0.24 & 0.53 & 32.159 & 0.000 & ** & 0.997 & 0.842 & 0.000 & ** & 0.892 & 0.000 \\
\hline EPDS10 & 0.07 & 0.29 & 0.06 & 0.28 & 0.07 & 0.30 & 0.08 & 0.34 & 1.112 & 0.343 & & & & & & & \\
\hline MIBS1 & 0.14 & 0.38 & 0.15 & 0.41 & 0.12 & 0.36 & 0.12 & 0.36 & 2.048 & 0.105 & & & & & & & \\
\hline MIBS2 & 0.56 & 0.67 & 0.56 & 0.72 & 0.58 & 0.71 & 0.55 & 0.71 & 0.304 & 0.822 & & & & & & & \\
\hline MIBS3 & 0.18 & 0.41 & 0.17 & 0.40 & 0.18 & 0.42 & 0.18 & 0.41 & 0.080 & 0.971 & & & & & & & \\
\hline MIBS4 & 0.05 & 0.28 & 0.04 & 0.25 & 0.04 & 0.23 & 0.05 & 0.31 & 0.748 & 0.523 & & & & & & & \\
\hline MIBS5 & 0.09 & 0.30 & 0.10 & 0.33 & 0.11 & 0.33 & 0.10 & 0.30 & 0.307 & 0.820 & & & & & & & \\
\hline MIBS6 & 0.58 & 0.73 & 0.50 & 0.68 & 0.57 & 0.73 & 0.48 & 0.67 & 4.562 & 0.003 & * & 0.080 & 0.992 & 0.023 & * & 0.095 & 0.948 \\
\hline MIBS7 & 0.07 & 0.32 & 0.06 & 0.30 & 0.07 & 0.33 & 0.05 & 0.28 & 0.795 & 0.497 & & & & & & & \\
\hline MIBS8 & 0.10 & 0.36 & 0.09 & 0.37 & 0.08 & 0.34 & 0.09 & 0.35 & 0.321 & 0.810 & & & & & & & \\
\hline MIBS9 & 0.06 & 0.25 & 0.06 & 0.30 & 0.08 & 0.35 & 0.06 & 0.31 & 1.273 & 0.282 & & & & & & & \\
\hline MIBS10 & 0.21 & 0.50 & 0.20 & 0.49 & 0.20 & 0.52 & 0.17 & 0.48 & 1.510 & 0.210 & & & & & & & \\
\hline
\end{tabular}

Regarding the cut-off score, the Kruskal-Wallis test showed no significant differences in the proportion of postpartum depression and postpartum pathological maternal bonding among the three Before COVID-19 groups and the During COVID-19 group (postpartum depression; 2017: 17.0\%; 2018: 14.2\%; 2019: 15.3\%; and 2020: $15.8 \% ; \chi^{2}=2.800, p=0.423$; postpartum pathological maternal bonding; $2017: 12.5 \% ; 2018: 12.9 \% ; 2019: 12.4 \%$; and $2020: 10.7 \% ; \chi^{2}=2.906, p=$ 0.406).

Furthermore, we also divided the During COVID-19 group into twelve subgroups according to month. To clarify the changes in scores during the COVID-19 pandemic and the relationships with the outbreak course, we compared the average sum scores for each factor of the EPDS and MIBS among these twelve subgroups with the number of the newly confirmed cases of COVID-19 in Tokyo and Kanagawa (Figs. 4) [42].

No significant changes in the sum score of each factor of the EPDS and MIBS were seen among these periods (EPDS anhedonia, $F=0.726, p=0.714, \eta^{2}=$ 0.007; EPDS anxiety, $F=0.838, p=0.602, \eta^{2}=0.008$; EPDS depression, $F=0.616, p=0.816, \eta^{2}=0.006$; MIBS lack of affection, $F=0.640, p=0.795, \eta^{2}=0.006$; MIBS anger and rejection, $F=0.625, p=0.809, \eta^{2}=0.006$ ), using the one-way ANOVA. With reference to Fig. 4 , the monthly changes of the sum score for each factor and the number of newly confirmed cases of COVID-19 seemed to be poorly related.

\section{Discussion}

To the best of our knowledge, this study was the first to examine the psychological response of postpartum mothers during the COVID-19 pandemic in a metropolitan area of Japan. The total EPDS and MIBS scores did not differ significantly among the three Before COVID-19 and the During COVID-19 groups. However, when the subscales of the EPDS were examined using MANOVA, the subscales related to anxiety factor were significantly higher in the During COVID-19 group, while the subscales related to depression and anhedonia factors, except for thoughts of self-harm, were lower in the During COVID-19 group.

\section{COVID-19 as a disaster}

Although an established definition of 'disaster' does not exist, disasters have been said to have three important characteristics. The first is a threat of harm or death to many people. The second is a disruption in social processes, services, and networks. And the third is an effect on mental and physical outcomes. The 
COVID-19 pandemic can be defined as a worldwide disaster [43]. Exposure to disasters has been associated with various mental health problems, including posttraumatic stress disorder, major depressive disorder, substance use disorder, and generalized anxiety disorder. Reportedly, women are less likely to be resilient during the post-disaster period than men, regardless of the type of disaster [44]. People living with children are also less likely to be resilient, perhaps because of their concerns and responsibilities toward their children. Longitudinal studies have shown that post-disaster mental health problems generally peak within a year and then improve, but in some people the symptoms persist [45]. However, COVID-19 is an ongoing disaster with no sign of a worldwide end. Therefore, mental health problems may become more serious in the future. The present study may serve as an important resource for clarifying the longitudinal course of postpartum maternal mental health during the COVID-19 pandemic.

\section{COVID-19-related anxiety}

Our survey revealed that during the COVID-19 pandemic, new mothers had higher levels of anxiety than those who gave birth before COVID-19. Pregnancy and childbirth can be stressful times for women even during normal circumstances; factors related to the COVID-19 pandemic may have further increased pregnancy- and childbirth-related anxiety. Previous articles have suggested that many people were worried about transmitting COVID-19 to their families, and a certain number of people exhibited pathological health anxiety characterized by an excessive fear of COVID-19 [10, 46]. Furthermore, a recent study revealed that COVID-19-related health worries may exacerbate mental health problems among pregnant women [47]. In addition, the unpredictability and uncertainty of the COVID-19 pandemic may also increase anxiety. Given the high degree of transmissibility and potential lethality of COVID-19, we believe that anxiety levels increased because of concerns over transmitting COVID-19 to their newborn child and cognitive changes arising from alertness to potential threats.

Furthermore, COVID-19-related physical distancing might have led to social isolation, limited access to basic services, and decreased family and social support [30, 33]. In Japan, many women have been greatly influenced by the COVID-19 pandemic during the course of their pregnancy and postpartum period, including changes in the place of delivery (8\%), the cancellation of planned formal or informal support (23\%), and the cancellation of parenting classes (79\%) $[33,34]$. Our investigation might reflect COVID-19-related health worries and a lack of social support because of the COVID-19 pandemic. The increase in anxiety among peripartum women during the COVID-19 pandemic was consistent with the results of a previous meta-analysis [26]. Anxiety during the pregnancy period can reportedly have negative effects on a child's mental development, internalizing problems, and cognitive function [48, 49]. Therefore, the current anxiety of perinatal women must be better understood, and an integrated approach to providing care is needed.

\section{Relationships among anxiety, anhedonia, and depression}

Our results showed that depression and anhedonia were lower during the COVID-19 pandemic, and this result seems to be inconsistent with previous studies $[26,27]$. However, hypervigilance, which is a known feature of post-traumatic stress disorder, might be the key to interpreting these results [43, 44]. Hypervigilance is a physiological and cognitive state of persistent hyperarousal and alertness against potential threats that allows a threat to be easily detected and a quick response to be made in potentially dangerous situations [50]. Physiological factors (i.e., increased alertness) are said to be mediated by the activation of the neuroendocrine stress system [51]. Hyperarousal and alertness might be the opposite states of depression and anhedonia. If anxiety and stress regarding the threat posed by COVID-19 cause hypervigilance, then the relative reduction in depression and anhedonia might also reflect the influence of hypervigilance [50]. In the present study, the results were considered to be consistent with mental health during a disaster. The EPDS results appear to be largely interpretable based on the concept of hypervigilance with the possible exception of Item 7, which may be slightly inconsistent. Item 7 consists of insomnia, which should be exacerbated during the COVID-19 pandemic because insomnia is one of the characteristics of hypervigilance [50]. Other factors may have an impact on the improvement of apparent insomnia.

When hypervigilance becomes a chronic condition characterized by sustained activation and failure to deregulate warning responses, people may experience impairments in their quality of life, such as the exacerbation of depression and anhedonia. A previous longitudinal study using path analysis attempted to clarify the relationship among anhedonia, anxiety, and depression, and the results suggested that anxiety led to anhedonia and then to depression over time [52]. This study suggests that the chronic impact of the COVID-19 pandemic may lead to an eventual reverse in the downward trend of anhedonia and depression. The outcome will depend on multiple factors and the presence of resilience as a normal coping and adaption; thus, the changes associated with chronic courses should be followed continuously [53].

Of note, suicidal ideation (EPDS item 10), which is a component of the "EPDS Depression" factor, did not differ significantly between the Before and During COVID-19 groups, although the total depressive symptoms decreased during COVID-19. The suicide rate of peripartum women in Japan is estimated to be 8.7 per births of 100,000, which is higher than that in Western countries [54]. Furthermore, violent methods of suicide attempts are associated with critical perinatal outcomes [55]. Economic issues associated with the COVID-19 pandemic may also contribute to the exacerbation of suicidal ideation. Suicide ideation must be carefully considered because suicide attempts can lead to fatal consequences for both mother and child.

\section{Above cut-off}

The prevalence of postpartum depression in Western countries is estimated to be $13 \%-19 \%$ [56]. In Japan, a recent meta-analysis involving one hundred thousand Japanese women found that the prevalence of postpartum depression at one month after childbirth was 14.3\% [57]. No significant difference in the prevalence of postpartum depression was seen between the three Before and the During COVID-19 groups, and the prevalence was almost equal to that reported in a recent Japanese meta-analysis [57]. As of December 2020, our results suggested that COVID-19 had little effect on the prevalence of postpartum depression and pathological maternal bonding.

\section{Maternal bonding}

MANOVA revealed only the score for MIBS Items 6 was significant difference between the four groups, however, post hoc comparison showed there were no significant difference in 2018 vs. 2020. Therefore, this difference does not seem to be important clinically. Previous studies have suggested that anxiety was 
negatively associated with mother-to-infant bonding and that depressive symptoms were predictors of future bonding [58, 59]. Since qualitative changes in the EPDS subscales have occurred, maternal bonding may change in the future. Moreover, as the COVID-19 pandemic becomes increasingly chronic, it may come to have a negative influence on maternal bonding.

\section{Changes in mean scores of EPDS and MIBS throughout the COVID-19 pandemic}

Figure 4 show the changes in the EPDS and MIBS scores throughout the COVID-19 pandemic. The increase in daily reported number of confirmed COVID-19 patients affected the psychological distress of the postpartum mothers and also caused social restrictions. A previous study in Wuhan showed that the prevalence of perinatal depression increased more as the COVID-19 pandemic worsened more [60]. However, our results showed that there was no changes of the average sum scores of the EPDS and MIBS. The third wave of COVID-19 cases has arrived in Japan since December 2020, and a state of emergency was issued again in January 2021 [42]. Because of the effects of chronic stress and the state of emergency, it is essential to observe future trends.

\section{Community-based integrated care system and implementation}

Possible barriers to the use of medical services must be considered. Middle-aged adults, women, and those who have experienced panic symptoms reportedly use mental health services less frequently after disasters [43]. In a UK study, women who died by suicide during the perinatal period were less likely to have contacted any psychiatric services before their suicide, compared with non-perinatal women [61]. In addition, because of the COVID-19 pandemic, mothers are being forced to restrict the use of medical services. During the COVID-19 pandemic, pregnant and postpartum women have experienced the cancellation of planned social support, and young people who had mental health problems were not able to access mental health support [34,62].

Perceived supports provided by health care staff can be regarded as protective factors against stress-related symptoms [25]. Besides, a community-based integrated approach, including suicide prevention, may contribute to the maintenance of peripartum mothers' mental health [63, 64]. Our results suggest the need to strengthen both general public health interventions and mental health care services during the COVID-19 pandemic. Suicide prevention for perinatal women is also important. Online support may be an optimal and effective option to assess each mother's psychological and social needs, and such support might reduce psychological distress and prevent adverse effects on long-term mental health $[33,63,65,66]$.

Previous research has shown that an important factor associated with postpartum depression during the COVID-19 pandemic was immigration status [22]. Foreign nationals are unevenly distributed in metropolitan areas in Japan, and foreign nationals account for about four percent of the population in eastern Kanagawa [67]. We revealed that foreign nationals are less likely to contact appropriate services for mental illness; therefore, a community-based integrated care system that is accessible to foreign nationals during the perinatal period is also needed [68]. Further research is required to clarify individual responses and resilience, taking into account social, economic and cultural contexts.

\section{Strengths and Limitations}

Many of the studies that are currently being published have been conducted using online surveys because of convenience and COVID-19 precautions [9, 25, 34]. Online surveys inevitably encounter the problem of spoofing, which is detrimental to an accurate understanding of mental health. On the other hand, the screening questionnaire used in our study was completed face-to-face at the hospital by nurses or midwives. Furthermore, although a small number of missing data were excluded, this was a retrospective study of almost all the mothers who gave birth at one regional core medical institution. As a result, our study had a reduced sampling bias and might be a valuable resource for understanding the actual state of perinatal mental health in a metropolitan area of Japan during the COVID-19 pandemic. Moreover, one strength of this study is that the impact of COVID-19 was clarified by comparing the scores obtained during the pandemic with those obtained in a control group that reflected the situation before the COVID-19 pandemic [34].

Some limitations of this study should be noted. First, the causal relationship between the present results and the COVID-19 pandemic could not be established because the study consisted of a retrospective chart review. However, a yearly comparison strongly suggested that the changes in the mean scores for each item of the EPDS were caused by the COVID-19 pandemic. Second, we relied on information derived from self-reported measures, and the participants consisted of mothers visiting a single hospital. Third, this study did not examine potentially confounding factors affecting postpartum mental health, such as the current economic situation, social support, cultural context, and feelings toward pregnancy $[22,69]$. Further studies to address these potentially confounding factors are needed.

\section{Conclusions}

This study demonstrated the current status of post-partum mental health during the COVID-19 pandemic, compared with the prior situation. Even in Japan where the number of newly confirmed COVID-19 cases is relatively small in the world, the COVID-19 pandemic has a negative impact on the mental health in postpartum mothers. Anxiety was significantly higher while anhedonia and depression were significantly lower during the COVID-19 pandemic, suggesting a state of hypervigilance. Our investigation may reflect COVID-19-related health concerns and the lack of social support caused by the COVID-19 pandemic. An optimal community-based integrated mental health care system for postpartum mothers is also required in the COVID-19 era. Further research is needed to clarify individual responses and resilience, taking into account social, economic and cultural contexts, and the long-term effects of the COVID-19 pandemic on postpartum mental health.

\section{Abbreviations}

COVID-19: coronavirus disease 2019; EPDS: Edinburgh Postnatal Depression Scale; MIBS: Mother-to-Infant Bonding Scale; STAI: State-Trait Anxiety Inventory; MANOVA: the multivariate analysis of variance. 


\section{Declarations}

\section{Ethics approval and consent to participate}

The study protocol was approved by the Ethics Committees of Saiseikai Yokohamashi Tobu Hospital (20200129). Informed consent was obtained in the form of opt-out on a website.

\section{Consent for publication}

Informed consent was obtained in the form of opt-out on a website.

\section{Availability of data and materials}

The data sets used and /or analyzed during the present study are available from the corresponding author upon reasonable request.

\section{Competing interests}

The authors declare that they have no competing interests.

\section{Funding}

This work was supported by a Health Labour Sciences Research Grant (19GC1015) to T.N. and N.T. The funding body had no role in the study design, data collection, analyses, interpretation of data, or writing of the manuscript.

\section{Authors' contributions}

T. and T.N. conceived the idea and methodology for this study. N.T., Y.Ai., K.F., M.IW., T.U., M.It., Y.Ak., and M.M. were involved in the conceptualization level of the study. Y.T., Y.Ai., and K.F. collected the data. Y.T., N.T., and T.N. analyzed the data. Y. T. and T.N. wrote the first draft of the manuscript. All the authors contributed to the preparation of the final manuscript and approved its submission.

\section{Acknowledgements}

We thank all the study participants and the staff of the Obstetrics and Gynecology Department at Saiseikai Yokohamashi Tobu Hospital.

\section{References}

1. Wang C, Horby PW, Hayden FG, Gao GF: A novel coronavirus outbreak of global health concern. The Lancet 2020, 395(10223):470-473. http://doi.org/10.1016/s0140-6736(20)30185-9.

2. Nishiura H, Jung SM, Linton NM, Kinoshita R, Yang Y, Hayashi K, Kobayashi T, Yuan B, Akhmetzhanov AR: The Extent of Transmission of Novel Coronavirus in Wuhan, China, 2020. J Clin Med 2020, 9(2). http://doi.org/10.3390/jcm9020330.

3. Cucinotta D, Vanelli M: WHO Declares COVID-19 a Pandemic. Acta Biomed 2020, 91(1):157-160. http://doi.org/10.23750/abm.v91i1.9397.

4. Cabinet Secretariat. Japan's Response to the Novel Coronavirus Disease: Declaration of a State of Emergency. 2020. Available from https://corona.go.jp/en/. Accessed 24 June 2021.

5. Pierce M, Hope H, Ford T, Hatch S, Hotopf M, John A, Kontopantelis E, Webb R, Wessely S, McManus S et al: Mental health before and during the COVID-19 pandemic: a longitudinal probability sample survey of the UK population. The Lancet Psychiatry 2020, 7(10):883-892. http://doi.org/10.1016/s22150366(20)30308-4.

6. Guessoum SB, Lachal J, Radjack R, Carretier E, Minassian S, Benoit L, Moro MR: Adolescent psychiatric disorders during the COVID-19 pandemic and lockdown. Psychiatry Res 2020, 291:113264. http://doi.org/10.1016/j.psychres.2020.113264.

7. Cao W, Fang Z, Hou G, Han M, Xu X, Dong J, Zheng J: The psychological impact of the COVID-19 epidemic on college students in China. Psychiatry Res 2020, 287:112934. http://doi.org/10.1016/j.psychres.2020.112934.

8. Qiu J, Shen B, Zhao M, Wang Z, Xie B, Xu Y: A nationwide survey of psychological distress among Chinese people in the COVID-19 epidemic: implications and policy recommendations. Gen Psychiatr 2020, 33(2):e100213. http://doi.org/10.1136/gpsych-2020-100213.

9. Salari N, Hosseinian-Far A, Jalali R, Vaisi-Raygani A, Rasoulpoor S, Mohammadi M, Rasoulpoor S, Khaledi-Paveh B: Prevalence of stress, anxiety, depression among the general population during the COVID-19 pandemic: a systematic review and meta-analysis. Global Health 2020, $16(1): 57$. http://doi.org/10.1186/s12992-020-00589-w.

10. Barzilay R, Moore TM, Greenberg DM, DiDomenico GE, Brown LA, White LK, Gur RC, Gur RE: Resilience, COVID-19-related stress, anxiety and depression during the pandemic in a large population enriched for healthcare providers. Transl Psychiatry 2020, 10(1):291. http://doi.org/10.1038/s41398-02000982-4.

11. Li J, Yang Z, Qiu H, Wang Y, Jian L, Ji J, Li K: Anxiety and depression among general population in China at the peak of the COVID-19 epidemic. World Psychiatry 2020, 19(2):249-250. http://doi.org/10.1002/wps.20758.

12. Liu N, Zhang F, Wei C, Jia Y, Shang Z, Sun L, Wu L, Sun Z, Zhou Y, Wang Y et al: Prevalence and predictors of PTSS during COVID-19 outbreak in China hardest-hit areas: Gender differences matter. Psychiatry Res 2020, 287:112921. http://doi.org/10.1016/j.psychres.2020.112921. 
13. Ueda M, Stickley A, Sueki H, Matsubayashi T: Mental health status of the general population in Japan during the COVID-19 pandemic. Psychiatry Clin Neurosci 2020, 74(9):505-506. http://doi.org/10.1111/pcn.13105.

14. Gavin NI, Gaynes BN, Lohr KN, Meltzer-Brody S, Gartlehner G, Swinson T: Perinatal depression: a systematic review of prevalence and incidence. Obstet Gynecol 2005, 106(5 Pt 1):1071-1083. http://doi.org/10.1097/01.AOG.0000183597.31630.db.

15. Vesga-Lopez O, Blanco C, Keyes K, Olfson M, Grant BF, Hasin DS: Psychiatric disorders in pregnant and postpartum women in the United States. Arch Gen Psychiatry 2008, 65(7):805-815. http://doi.org/10.1001/archpsyc.65.7.805.

16. Howard LM, Molyneaux E, Dennis C-L, Rochat T, Stein A, Milgrom J: Non-psychotic mental disorders in the perinatal period. The Lancet 2014, 384(9956):1775-1788. http://doi.org/10.1016/s0140-6736(14)61276-9.

17. Stein A, Pearson RM, Goodman SH, Rapa E, Rahman A, McCallum M, Howard LM, Pariante CM: Effects of perinatal mental disorders on the fetus and child. The Lancet 2014, 384(9956):1800-1819. http://doi.org/10.1016/s0140-6736(14)61277-0.

18. Caparros-Gonzalez RA, Ganho-Avila A, Torre-Luque A: The COVID-19 Pandemic Can Impact Perinatal Mental Health and the Health of the Offspring. Behav Sci (Basel) 2020, 10(11). http://doi.org/10.3390/bs10110162.

19. Brown S: Perinatal mental health and the COVID-19 pandemic. World Psychiatry 2020, 19(3):333-334. http://doi.org/10.1002/wps.20779.

20. Wu Y, Zhang C, Liu H, Duan C, Li C, Fan J, Li H, Chen L, Xu H, Li X et al: Perinatal depressive and anxiety symptoms of pregnant women during the coronavirus disease 2019 outbreak in China. Am J Obstet Gynecol 2020, 223(2):240 e241-240 e249. http://doi.org/10.1016/j.ajog.2020.05.009.

21. Durankus F, Aksu E: Effects of the COVID-19 pandemic on anxiety and depressive symptoms in pregnant women: a preliminary study. J Matern Fetal Neonatal Med 2020:1-7. http://doi.org/10.1080/14767058.2020.1763946.

22. Liang P, Wang Y, Shi S, Liu Y, Xiong R: Prevalence and factors associated with postpartum depression during the COVID-19 pandemic among women in Guangzhou, China: a cross-sectional study. BMC Psychiatry 2020, 20(1):557. http://doi.org/10.1186/s12888-020-02969-3.

23. Ayaz R, Hocaoglu M, Gunay T, Yardimci OD, Turgut A, Karateke A: Anxiety and depression symptoms in the same pregnant women before and during the COVID-19 pandemic. J Perinat Med 2020, 48(9):965-970. http://doi.org/10.1515/jpm-2020-0380.

24. Moyer CA, Compton SD, Kaselitz E, Muzik M: Pregnancy-related anxiety during COVID-19: a nationwide survey of 2740 pregnant women. Arch Womens Ment Health 2020. http://doi.org/10.1007/s00737-020-01073-5.

25. Ostacoli L, Cosma S, Bevilacqua F, Berchialla P, Bovetti M, Carosso AR, Malandrone F, Carletto S, Benedetto C: Psychosocial factors associated with postpartum psychological distress during the Covid-19 pandemic: a cross-sectional study. BMC Pregnancy Childbirth 2020, 20(1):703. http://doi.org/10.1186/s12884-020-03399-5.

26. Hessami K, Romanelli C, Chiurazzi M, Cozzolino M: COVID-19 pandemic and maternal mental health: a systematic review and meta-analysis. J Matern Fetal Neonatal Med 2020:1-8. http://doi.org/10.1080/14767058.2020.1843155.

27. Yan H, Ding Y, Guo W: Mental Health of Pregnant and Postpartum Women During the Coronavirus Disease 2019 Pandemic: A Systematic Review and Meta-Analysis. Front Psychol 2020, 11:617001. http://doi.org/10.3389/fpsyg.2020.617001.

28. Lopez-Morales H, Del Valle MV, Canet-Juric L, Andres ML, Galli JI, Poo F, Urquijo S: Mental health of pregnant women during the COVID-19 pandemic: A longitudinal study. Psychiatry Res 2021, 295:113567. http://doi.org/10.1016/j.psychres.2020.113567.

29. Brockington I: Maternal rejection of the young child: present status of the clinical syndrome. Psychopathology 2011, 44(5):329-336. http://doi.org/10.1159/000325058.

30. Ohara M, Nakatochi M, Okada T, Aleksic B, Nakamura Y, Shiino T, Yamauchi A, Kubota C, Morikawa M, Murase S et al: Impact of perceived rearing and social support on bonding failure and depression among mothers: A longitudinal study of pregnant women. J Psychiatr Res 2018, 105:71-77.

http://doi.org/10.1016/j.jpsychires.2018.09.001

31. Takubo Y, Nemoto T, Obata Y, Baba Y, Yamaguchi T, Katagiri N, Tsujino N, Kitamura T, Mizuno M: Effectiveness of Kangaroo Care for a Patient with Postpartum Depression and Comorbid Mother-Infant Bonding Disorder. Case Rep Psychiatry 2019, 2019:9157214. http://doi.org/10.1155/2019/9157214.

32. Oskovi-Kaplan ZA, Buyuk GN, Ozgu-Erdinc AS, Keskin HL, Ozbas A, Moraloglu Tekin O: The Effect of COVID-19 Pandemic and Social Restrictions on Depression Rates and Maternal Attachment in Immediate Postpartum Women: a Preliminary Study. Psychiatr Q 2020. http://doi.org/10.1007/s11126020-09843-1.

33. Haruna M, Nishi D: Perinatal mental health and COVID-19 in Japan. Psychiatry Clin Neurosci 2020, 74(9):502-503. http://doi.org/10.1111/pcn.13091.

34. Matsushima M, Horiguchi H: The COVID-19 Pandemic and Mental Well-Being of Pregnant Women in Japan: Need for Economic and Social Policy Interventions. Disaster Med Public Health Prep 2020:1-6. http://doi.org/10.1017/dmp.2020.334.

35. Kanagawa Prefectural Government: Epidemiological Surveillance of Infectious Diseases in Kanagawa Prefecture (in Japanese). 2021. Available from https://www.pref.kanagawa.jp/osirase/1369/. Accessed 24 June 2021.

36. Cox JL, Holden JM, Sagovsky R: Detection of postnatal depression. Development of the 10-item Edinburgh Postnatal Depression Scale. Br J Psychiatry 1987, 150:782-786. http://doi.org/10.1192/bjp.150.6.782.

37. Okano TMM, Masuji F, Tamaki R, Nomura J, Miyaoka H, Kitamura K: Validation and reliability of a Japanese version of the EPDS. Arch Psychiatr Diagn Clin Eval 1996, 7:525-533.

38. Taylor A, Atkins R, Kumar R, Adams D, Glover V: A new Mother-to-Infant Bonding Scale: links with early maternal mood. Arch Womens Ment Health 2005, 8(1):45-51. http://doi.org/10.1007/s00737-005-0074-z.

39. Yoshida K, Yamashita H, Conroy S, Marks M, Kumar C: A Japanese version of Mother-to-Infant Bonding Scale: factor structure, longitudinal changes and links with maternal mood during the early postnatal period in Japanese mothers. Arch Womens Ment Health 2012, 15(5):343-352.

http://doi.org/10.1007/s00737-012-0291-1. 
40. Matsumura K, Hamazaki K, Tsuchida A, Kasamatsu H, Inadera H, Japan E, Children's Study G: Factor structure of the Edinburgh Postnatal Depression Scale in the Japan Environment and Children's Study. Sci Rep 2020, 10(1):11647. http://doi.org/10.1038/s41598-020-67321-x.

41. Matsunaga A, Takauma F, Tada K, Kitamura T: Discrete category of mother-to-infant bonding disorder and its identification by the Mother-to-Infant Bonding Scale: A study in Japanese mothers of a 1-month-old. Early Hum Dev 2017, 111:1-5. http://doi.org/10.1016/j.earlhumdev.2017.04.008.

42. Ministry of Health, Labour and Welfare: Visualizing the data: information on COVID-19 infections. 2021. Available from https://covid19.mhlw.go.jp/en/. Accessed 24 June 2021.

43. Goldmann E, Galea S: Mental health consequences of disasters. Annu Rev Public Health 2014, 35:169-183. http://doi.org/10.1146/annurev-publhealth032013-182435.

44. Norris FH, Friedman MJ, Watson PJ, Byrne CM, Diaz E, Kaniasty K: 60,000 disaster victims speak: Part I. An empirical review of the empirical literature, 1981-2001. Psychiatry 2002, 65(3):207-239. http://doi.org/10.1521/psyc.65.3.207.20173.

45. Norris FH, Friedman MJ, Watson PJ: 60,000 disaster victims speak: Part II. Summary and implications of the disaster mental health research. Psychiatry 2002, 65(3):240-260. http://doi.org/10.1521/psyc.65.3.240.20169.

46. Tyrer P: COVID-19 health anxiety. World Psychiatry 2020, 19(3):307-308. http://doi.org/10.1002/wps.20798.

47. Liu CH, Erdei C, Mittal L: Risk factors for depression, anxiety, and PTSD symptoms in perinatal women during the COVID-19 Pandemic. Psychiatry Res 2021, 295:113552. http://doi.org/10.1016/j.psychres.2020.113552.

48. Field T: Prenatal anxiety effects: A review. Infant Behav Dev 2017, 49:120-128. http://doi.org/10.1016/j.infbeh.2017.08.008.

49. Tan HK, Goh SKY, Tsotsi S, Bruntraeger M, Chen HY, Broekman B, Tan KH, Chong YS, Meaney MJ, Qiu A et al: Maternal antenatal anxiety and electrophysiological functioning amongst a sub-set of preschoolers participating in the GUSTO cohort. BMC Psychiatry 2020, 20 (1):62. http://doi.org/10.1186/s12888-020-2454-3.

50. Kleshchova O, Rieder JK, Grinband J, Weierich MR: Resting amygdala connectivity and basal sympathetic tone as markers of chronic hypervigilance. Psychoneuroendocrinology 2019, 102:68-78. http://doi.org/10.1016/j.psyneuen.2018.11.036.

51. Pole N: The psychophysiology of posttraumatic stress disorder: a meta-analysis. Psychol Bull 2007, 133(5):725-746. http://doi.org/10.1037/00332909.133.5.725.

52. Winer ES, Bryant J, Bartoszek G, Rojas E, Nadorff MR, Kilgore J: Mapping the relationship between anxiety, anhedonia, and depression. J Affect Disord 2017, 221:289-296. http://doi.org/10.1016/j.jad.2017.06.006.

53. Norris FH, Tracy M, Galea S: Looking for resilience: understanding the longitudinal trajectories of responses to stress. Soc Sci Med 2009, 68(12):21902198. http://doi.org/10.1016/j.socscimed.2009.03.043.

54. Takeda S: Suicide prevention in perinatal periods. Perinatal Med 2017, 47(5):623-627.

55. Shigemi D, Ishimaru M, Matsui H, Fushimi K, Yasunaga H: Suicide attempts during pregnancy and perinatal outcomes. J Psychiatr Res 2021, 133:101105. http://doi.org/10.1016/j.jpsychires.2020.12.024.

56. O'Hara MW, McCabe JE: Postpartum depression: current status and future directions. Annu Rev Clin Psychol 2013, 9:379-407. http://doi.org/10.1146/annurev-clinpsy-050212-185612.

57. Tokumitsu K, Sugawara N, Maruo K, Suzuki T, Shimoda K, Yasui-Furukori N: Prevalence of perinatal depression among Japanese women: a metaanalysis. Ann Gen Psychiatry 2020, 19:41. http://doi.org/10.1186/s12991-020-00290-7.

58. Kasamatsu H, Tsuchida A, Matsumura K, Shimao M, Hamazaki K, Inadera H, Japan E, Children's Study G: Understanding the relationship between postpartum depression one month and six months after delivery and mother-infant bonding failure one-year after birth: results from the Japan Environment and Children's study (JECS). Psychol Med 2020, 50(1):161-169. http://doi.org/10.1017/S0033291719002101.

59. Motegi T, Watanabe Y, Fukui N, Ogawa M, Hashijiri K, Tsuboya R, Sugai T, Egawa J, Araki R, Haino K et al: Depression, Anxiety and Primiparity are Negatively Associated with Mother-Infant Bonding in Japanese Mothers. Neuropsychiatr Dis Treat 2020, 16:3117-3122. http://doi.org/10.2147/NDT.S287036.

60. Sun G, Wang Q, Lin Y, Li R, Yang L, Liu X, Peng M, Wang H, Yang X, Ren W et al: Perinatal Depression of Exposed Maternal Women in the COVID-19 Pandemic in Wuhan, China. Front Psychiatry 2020, 11:551812. http://doi.org/10.3389/fpsyt.2020.551812.

61. Khalifeh H, Hunt IM, Appleby L, Howard LM: Suicide in perinatal and non-perinatal women in contact with psychiatric services: 15 year findings from a UK national inquiry. The Lancet Psychiatry 2016, 3(3):233-242. http://doi.org/10.1016/s2215-0366(16)00003-1.

62. Youngminds: Coronavirus: Impact on young people with mental health needs. 2020. Available from https://youngminds.org.uk/media/3904/coronavirusreport-summer-2020-final.pdf?dm_i=21A8,6YAN9,USS7D0,RZK45,1. Accessed 24 June 2021.

63. Shidhaye R, Madhivanan P, Shidhaye P, Krupp K: An Integrated Approach to Improve Maternal Mental Health and Well-Being During the COVID-19 Crisis. Front Psychiatry 2020, 11:598746. http://doi.org/10.3389/fpsyt.2020.598746.

64. Tachibana Y, Koizumi N, Mikami M, Shikada K, Yamashita S, Shimizu M, Machida K, Ito H: An integrated community mental healthcare program to reduce suicidal ideation and improve maternal mental health during the postnatal period: the findings from the Nagano trial. BMC Psychiatry 2020, $20(1): 389$.

http://doi.org/10.1186/s12888-020-02765-z.

65. Zhou X, Snoswell CL, Harding LE, Bambling M, Edirippulige S, Bai X, Smith AC: The Role of Telehealth in Reducing the Mental Health Burden from COVID19. Telemed J E Health 2020, 26(4):377-379. http://doi.org/10.1089/tmj.2020.0068.

66. Brooks SK, Weston D, Greenberg N: Psychological impact of infectious disease outbreaks on pregnant women: rapid evidence review. Public Health 2020, 189:26-36. http://doi.org/10.1016/j.puhe.2020.09.006. 
67. Immigration Services Agency of Japan: The statics of foreign residents in Jun 2020 (in Japanese). 2021. Available from http://www.moj.go.jp/housei/toukei/toukei_ichiran_touroku.html. Accessed 24 June 2021.

68. Takubo Y, Nemoto T, Iwai M, Kashima M, Yamaguchi E, Maruyama A, Miura S, Saito H, Tsujino N, Mizuno M: Demographic and clinical characteristics of foreign nationals accessing psychiatric services in Japan: a multicentre study in a metropolitan area. BMC Psychiatry 2020, 20(1):569. http://doi.org/10.1186/s12888-020-02951-z.

69. Baba S, Kimura T, Ikehara S, Honjo K, Eshak ES, Sato T, Iso H, Japan E, Children's Study G: Impact of intention and feeling toward being pregnant on postpartum depression: the Japan Environment and Children's Study (JECS). Arch Womens Ment Health 2020, 23(1):131-137. http://doi.org/10.1007/s00737-018-0938-7.

\section{Figures}

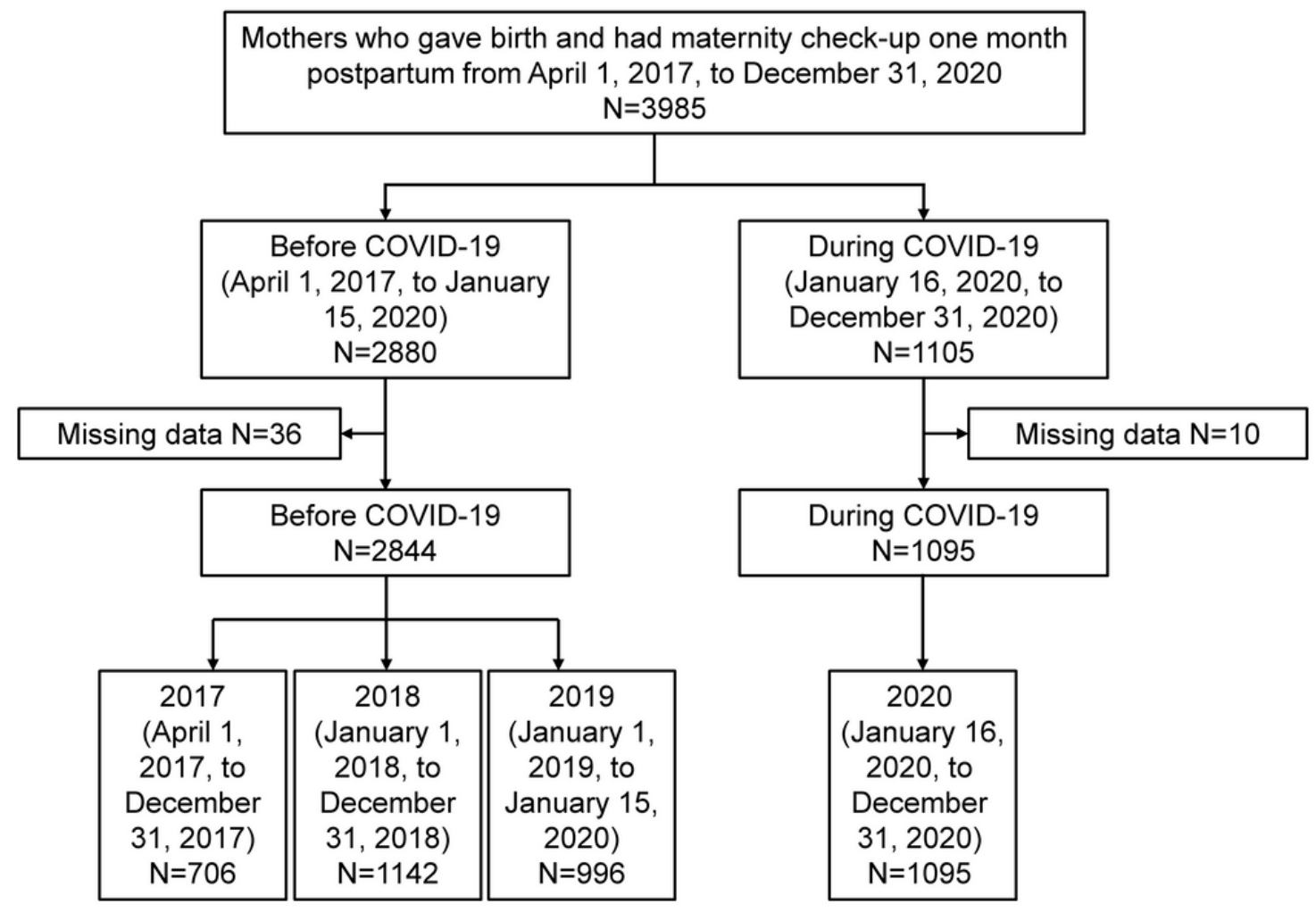

Figure 1

Study flow chart. 


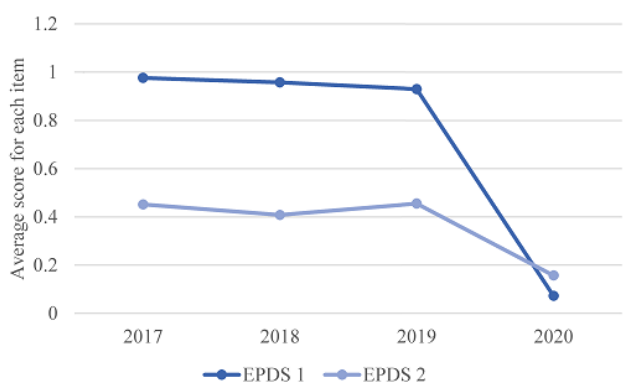

a

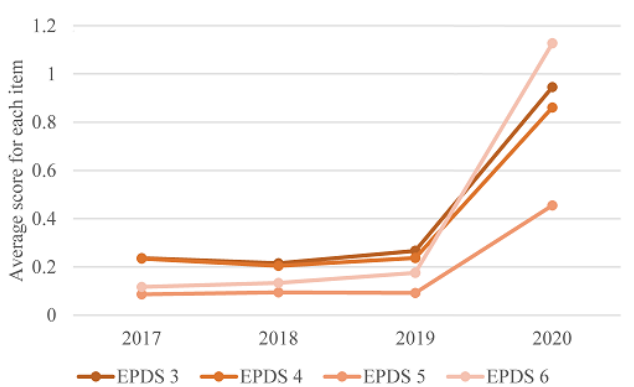

b

1.2

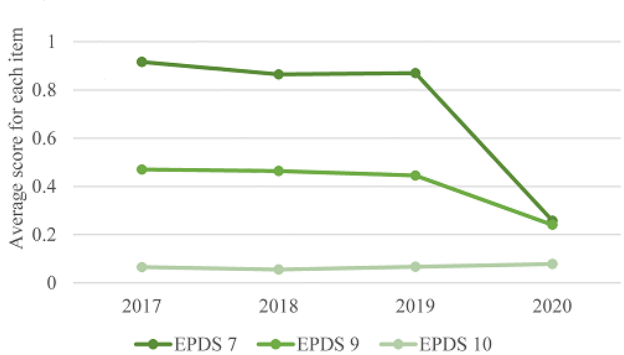

c

Figure 2

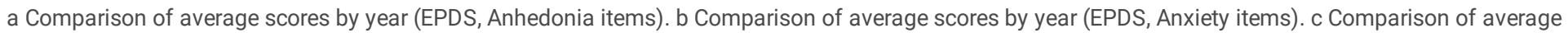

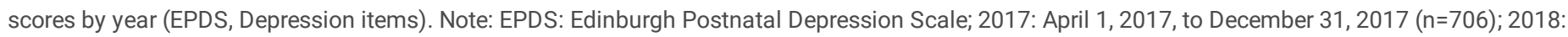

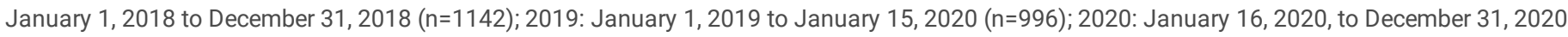
$(n=1095)$. 
a

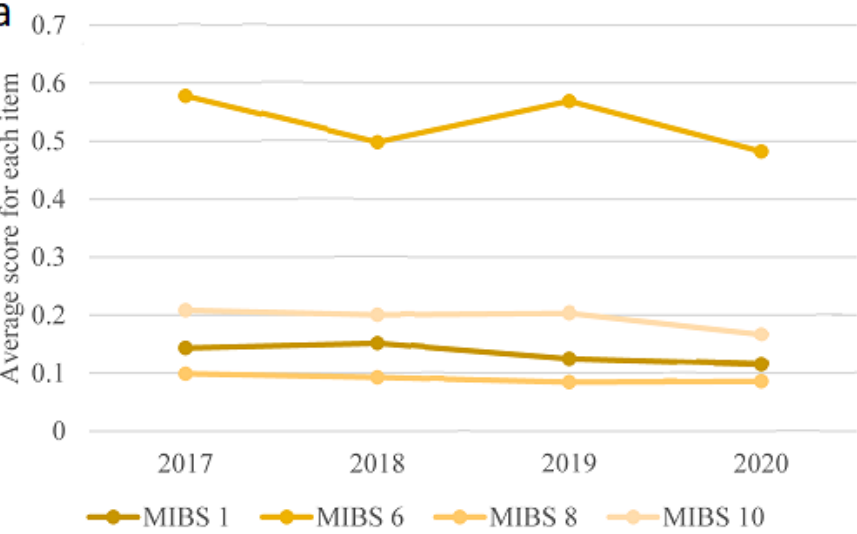

b

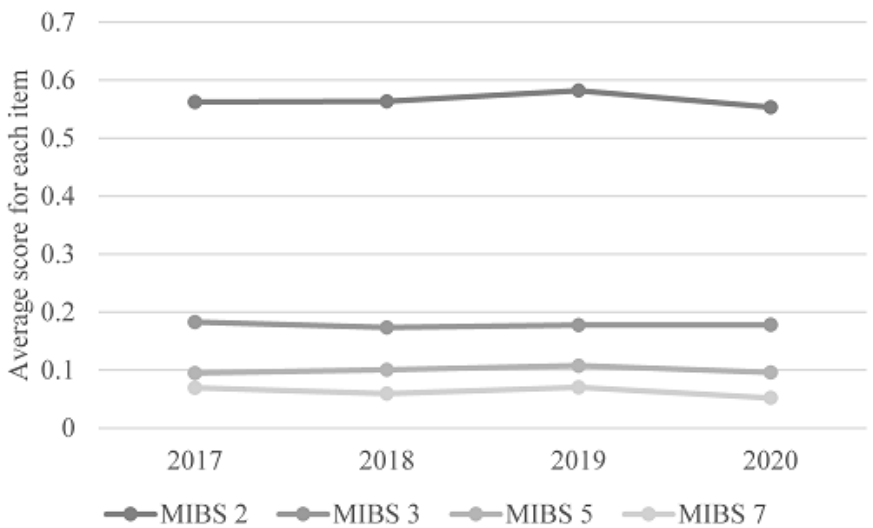

Figure 3

a Comparison of average scores by year (MIBS, Lack of affection items). b Comparison of average scores by year (MIBS, Anger and rejection items). Note: MIBS: Mother-to-Infant Bonding Scale; 2017: April 1, 2017, to December 31, 2017 ( $n=706)$; 2018: January 1, 2018 to December 31,2018 ( $n=1142$ ); 2019 : January 1, 2019 to January 15, 2020 ( $n=996) ; 2020$ : January 16, 2020, to December 31, $2020(n=1095)$. 


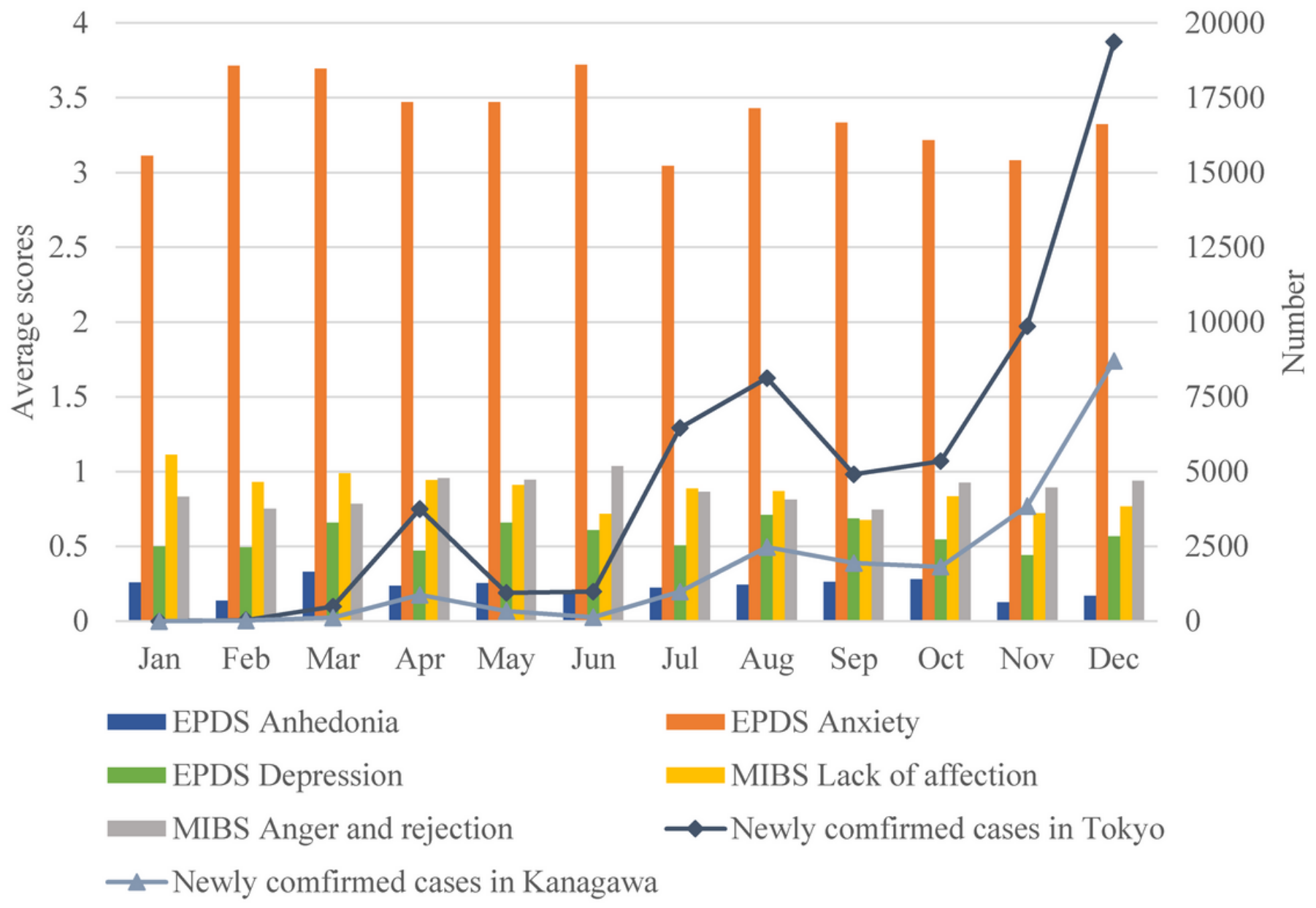

Figure 4

The newly confirmed cases of COVID-19 in Tokyo and Kanagawa and the average sum scores for each factor of the EPDS and MIBS during twelve time periods from January 16, 2020, to December 31, 2020. Surveillance data of COVID-19 were obtained from the official website of the Japanese Ministry of Health, Labour and Welfare (https://covid19.mhlw.go.jp/en/) [42]. Note: EPDS: Edinburgh Postnatal Depression Scale; MIBS: Mother-to-Infant Bonding Scale; EPDS Anhedonia: the sum of the EPDS scores for Items 1 and 2; EPDS Anxiety: the sum of the EPDS scores for Items 3, 4, 5, and 6 scores; EPDS Depression: the sum of the EPDS scores for Items 7, 9, and 10; MIBS Lack of affection: the sum of the MIBS scores for Items 1, 6, 8, and 10; MIBS Anger and rejection: the sum of the MIBS scores for Items 2, 3, 5, and 7. 